\title{
Avaliação da dureza e resistência ao impacto da resina acrílica para esclera de prótese ocular
}

\author{
ALESSANDRO RIBEIRO GONÇALVES , DOMICIO ROSENDO DA SILVA NETO* , MAXIMILIANO PIERO \\ NEISSER * , SIGMAR DE MELLO RODE **
}

\section{Resumo}

A resistência ao impacto e a dureza superficial de resinas acrílicas são muito importantes para o sucesso de próteses oculares devido a necessidade destas resistirem a choques mecânicos e de manterem o polimento por um maior período de tempo. Surgiu recentemente no mercado uma resina termo ativada específica para a confecção de escleras de próteses oculares (Clássico n. 3), não havendo informações na literatura sobre suas propriedades. A proposta desse trabalho foi de determinar a dureza e a resistência ao impacto dessa resina e comparar com uma resina incolor termo ativada (Clássico). Foram confeccionados 30 corpos-de-prova com $10 \mathrm{~mm}$ de largura por $50 \mathrm{~mm}$ de comprimento por $2 \mathrm{~mm}$ de espessura, sendo 15 de cada material, seguindo um ciclo de polimerização longo ( 9 horas a $74^{\circ} \mathrm{C}$ ). Após realizado o acabamento e polimento,os corposde-prova foram submetidos a ensaio de resistência ao impacto (EMIC - Equipamentos e sistemas de ensaios LTDA). Em seguida foi realizado o teste de dureza Knoop em um microdurômetro digital (Shimadzu - hmv-2000). Foram feitas 3 indentações em cada amostra e em seguida calculado o valor médio da dureza para cada corpo-de-prova. O valor médio de dureza Knoop da resina para esclera foi de 17,9 e para a incolor foi de 18,3 e de resistência ao impacto foi de $82 \mathrm{~J} / \mathrm{m}$ para a resina para esclera e de $88 \mathrm{~J} / \mathrm{m}$ para a resina incolor. Os resultados foram avaliados pelo teste " $t$ " de Student. Concluiu-se que não houve diferença estatística $(p>0,05)$ tanto para a dureza como para a resistência ao impacto entre as duas resinas.

\section{UNITERMOS}

Prótese ocular, resina acrílica termo-polimerizável, dureza superficial, resistência ao impacto.

GONÇALVES, A.R. et al. Avaluation of hardness and impact resistance of an acrilic resin for ocular prostheses. PGR-Pós-Grad Rev Fac. Odontol São José dos Campos, v.4, n.3, set./dez. 2001.

\section{Abstract}

The impact strenght and the superficial hardness of acrylic resins are very important for the success of ocular prostheses as these must resist to mechanical shocks and of maintain the polishing for a longer period of time. A heat-cured acrylic resin specific for the making of scleras for ocular prostheses (Clássico n. 3) appeared recently at the market, not having any information about its properties in the literature. The aim of this study was to determine the hardness and the resistance to the impact of this resin and to compare it with a heatcured acrylic colorless resin (Clássico). Thirty specimens were cured in $10 \times 50 \times 2 \mathrm{~mm}$ stone molds, being 15 of each material, following a long polymerization cycle $\left(74{ }^{\circ} \mathrm{C}\right.$ in water bath for 9 hours ), after which the finish and polishing were accomplished. The specimens were them submitted to impact strenght test (EMIC - Equipments and Systems of rehe-

\footnotetext{
*Aluno do curso de Pós-graduação em Odontologia (Nível Mestrado) - Área de concentração em Prótese Parcial Fixa - Faculdade de Odontologia de São José dos Campos - UNESP - 12.2450-000 - São José dos Campos - SP.

** Departamento de Materiais Odontológicos e Prótese - Faculdade de Odontologia de São José dos Campos - UNESP - 12.2450-000 - São José dos Campos - SP.
} 
arsals LTDA). Afterwards knoop hardness test was accomplished in a digital machine (Shimadzu - hmv-2000). Three indenting points were made in each sample and after calculated the medium hardness value for each specimen. The medium Knoop hardness value of the resin for sclera was of 17,9 and for the colorless it was 18,3.The medium impact resistance value was of $82 \mathrm{~J} / \mathrm{m}$ for the sclera resin and of $88 \mathrm{~J} / \mathrm{m}$ for the colorless resin. The results were appraised for the test " $t$ " of Student. The conclusion was that there was not statistic difference $(p>0,05)$ for both the hardness and impact strenght among these two resins.

\section{UNITERMS}

Ocular prostheses; heat-cured acrylic resin; superficial hardness; impact strenght.

\section{INTRODUÇÃO}

Há muitos anos já se fazia dentaduras para pacientes desdentados totais que necessitavam repor os dentes perdidos, para permitir uma melhor mastigação e devolver a estética, a harmonia facial, bem como a sua auto-estima. Antigamente estas próteses eram confeccionadas em vários materiais, iniciando pelo marfim e evoluindo até os dias atuais onde já se confecciona dentaduras em bases resilientes de resina acrílica, o que promove um maior conforto e aceitação por parte do paciente. Mesmo com toda essa evolução, o material mais usado para confecção de dentaduras nos dias de hoje, é o "polimetil metacrilato", uma resina acrílica que possui vários tipos de ativação, porém a mais comum ainda é através de ciclos de polimerização em banho maria.

Com o passar dos anos, a resina acrílica termicamente ativada, passou a ser utilizada também, como material principal no desenvolvimento de peças acrílicas para confecção de próteses bucomaxilo-faciais ${ }^{11}$, esses materiais foram evoluindo e chegaram à produção de uma resina acrílica específica para uso em esclera de prótese ocular.

Um dos principais problemas desse tipo de material, é que o fabricante não revela suas características físico-mecânicas, nem biológicas, deixando o Cirurgião-Dentista sem informações importantes para uma melhor utilização. Informações básicas de dureza superficial, alterações dimensionais após a presa, resistência ao impacto, liberação de monômero residual, dentre outras proprie- dades, se tornam indispensáveis para o protesista.

A resistência ao impacto mede a capacidade da prótese resistir a choques mecânicos e a dureza está diretamente relacionada com a capacidade de se manter o polimento do material por mais tempo e assim ser menos irritante à mucosa da cavidade ocular.

Robinson \& McCabe $^{10}$ (1993) avaliaram próteses totais feitas a partir de resinas acrílicas comuns e resinas que eram descritas como de alta resistência ao impacto. Os materiais foram avaliados quanto a resistência ao impacto e resistência a flexão. A resistência ao impacto se mostrou significativamente reduzida pela presença de pequenos defeitos de superfície $(<16 \mathrm{~mm})$, principalmente nas resinas denominadas de alta resistência. Nesses casos, a resistência ao impacto se mostrou apenas um pouco superior àquelas tidas como comuns.

Vallittu et al. ${ }^{12}$ (1995) testaram através do método Charpy a resistência ao impacto de corposde-prova de resina acrílica comum, em comparação com corpos-de-prova de resina acrílica reforçadas com fibras de aço de $1 \mathrm{~mm}$ de diâmetro e outro grupo reforçado por fibras de vidro. Os resultados mostraram um aumento da resistência ao impacto em todas os corpos-de-prova reforçados. Não foi encontrada diferença significativa na resistência entre os diferentes tipos de reforço.

Honorez et al. ${ }^{7}$ (1989) analisaram dois ciclos de polimerização para uma resina termo ativada recomendados pelo fabricante. Foram analisadas a resistência ao impacto e a dureza Brinnel. Observou-se que quando não se alcança o ponto de ebulição ocorre maior formação de monômero residual. As amostras se mostraram menos duras e mais resistentes. Quando usada água em ebulição, a porosidade aumentou e ocorreu diminuição da resistência ao impacto.

Diaz et al. ${ }^{4}$ (1999) testaram cinco tipos de materiais usados para confecção de restaurações provisórias com relação a dureza superficial. Amostras cilíndricas de resina bis-acryl (Integrity, Protemp Garant, Temphase) e de metil metacrilato (Jet, Temporary Bridge), foram fabricadas em número de 5 por material. Essas amostras foram polidas e armazenadas em saliva artificial a $37{ }^{\circ} \mathrm{C}$ por um total de 14 dias. A dureza Knoop foi medi- 
da 24 horas após a fabricação e depois de 14 dias. A dureza da maioria dos materiais (Integrity, Protemp Garant, Jet) diminuiu com o passar do tempo. Os materiais a base de bis-acryl exibiram maior dureza superficial, em comparação com as resinas a base de metil metacrilato (Jet, Temporary Bridge).

A escassa literatura a respeito das propriedades das resinas acrílicas para esclera de prótese ocular, nos levaram a realização de testes laboratoriais para esclarecer as características físico-mecânicas do material, estabelecendo assim, parâmetros para o uso pelo Cirurgião-Dentista, visto que a literatura encontrada, conota preocupação ape-

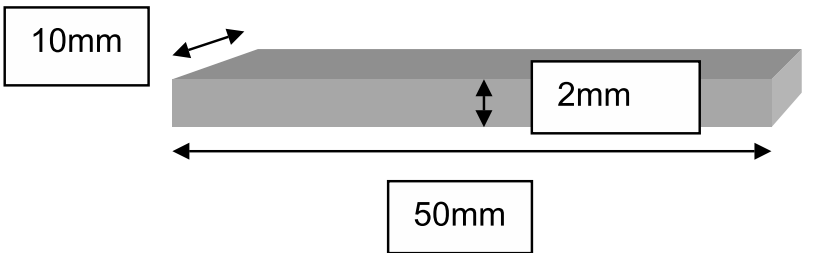

FIGURA 1 - dimensões da matriz plástica.

FIGURA 3 - mufla antes da prensagem. nas com as resinas para bases de dentaduras e/ou as resinas compostas.

\section{Material e Método}

Nesse estudo testou-se a resina acrílica para esclera termo ativada $n^{\circ} .3$ (Clássico) e a resina acrílica incolor termo ativada para base de dentaduras (Clássico) foi utilizada como controle. Para a confecção dos corpos-de-prova para os ensaios de Resistência ao Impacto e Dureza superficial, foi utilizada uma matriz plástica, com dimensões aproximadas de $10 \mathrm{~mm}$ de largura por $50 \mathrm{~mm}$ de comprimento por $2 \mathrm{~mm}$ de espessura (Figuras 1 e 2 ).

FIGURA 2 - matrizes plásticas.

FIGURA 4 - corpos-de-prova de resina para esclera de prótese ocular e resina incolor. 
Foram utilizadas seis muflas, três para cada resina, nas quais eram incluídas cinco matrizes em cada. Após a remoção das matrizes(Figura 3), eram prensadas a 1,5 toneladas e deixadas na prensa por 6 horas e depois levadas à polimerizadora onde ficavam em banho maria por mais 9 horas à $74^{\circ} \mathrm{C}^{1,2}$.

Após a confecção dos corpos-de-prova, 15 para cada resina, os mesmos foram lixados e polidos em suas faces superficiais, para que tivéssemos uma superfície plana e lisa (Figura 4). O teste de impacto Charpy foi realizado em uma máquina AIC1 EMIC (Equipamentos e sistemas de ensaios LTDA - PR). A máquina estava regulada com martelo de carga de $2,7 \mathrm{~J}$, e a resistência do ar foi medida antecipadamente, obtendo o valor de $0,03 \mathrm{~J}$. Os testes foram realizados da seguinte maneira: os corpos-de-prova foram posicionados na máquina e o pêndulo foi solto, levando com ele um ponteiro de arrasto, que marcaria o valor da energia dissipada, que seria convertido em valores de resistência ao impacto. Utilizando os mesmos corpos-deprova partidos ao meio, transformados agora em 30 amostras, realizamos o ensaio de dureza knoop, que foi realizado em um microdurômetro digital modelo hmv-2000 (Shimadzu). Foram feitas três indentações por corpo-de-prova, soltando o diamante Knoop com 25g de carga por 10s na superfície das amostras, fazendo as medições com microscópio com aumento de 200 vezes. Dos valores obtidos calculou-se a média de dureza superficial para cada espécime.

\section{Resultados}

Os resultados estão apresentados nas Tabelas 1 e 2 e nas figuras 5 e 6 .

Tabela 1 - Valores, média e desvio padrão obtidos no ensaio de resistência ao Impacto em J/m:

\begin{tabular}{|ccc|}
\hline Resistência ao Impacto & Resina Incolor & Resina para Esclera \\
\hline 1 & 73,14 & 73,14 \\
\hline 2 & 53,71 & 82,86 \\
\hline 4 & 14,76 & 82,86 \\
\hline 5 & 63,68 & 63,38 \\
\hline 6 & 73,14 & 82,86 \\
\hline 7 & 91,2 & 74,68 \\
\hline 8 & 96,25 & 101,3 \\
\hline 9 & 91,2 & 81,05 \\
\hline 10 & 129,23 & 73,22 \\
\hline 11 & 114,43 & 91,2 \\
\hline 12 & 101,3 & 91,2 \\
\hline 13 & 101,3 & 86,1 \\
\hline 14 & 106 & 81,05 \\
\hline 15 & 110,45 & 91,2 \\
\hline Média & 101,3 & 74,68 \\
\hline Desvio Padrão & 88,07 & 82,05 \\
\hline
\end{tabular}


Tabela 2 - Valores, média e desvio padrão obtidos no ensaio de dureza Knoop:

\begin{tabular}{|c|c|c|}
\hline Dureza Knoop & Resina Incolor & Resina para Esclera \\
\hline 1 & 19,9 & 20 \\
\hline 2 & 18,1 & 18,7 \\
\hline 3 & 18,1 & 14,4 \\
\hline 4 & 18,6 & 15,3 \\
\hline 5 & 19 & 17,8 \\
\hline 6 & 19 & 17,6 \\
\hline 7 & 18,6 & 15,8 \\
\hline 8 & 15,8 & 15,1 \\
\hline 9 & 20,3 & 17,4 \\
\hline 10 & 16,3 & 17,4 \\
\hline 11 & 18,1 & 18,5 \\
\hline 12 & 14,5 & 20 \\
\hline 13 & 15,9 & 18,6 \\
\hline 14 & 22,1 & 18,2 \\
\hline 15 & 17,3 & 17,4 \\
\hline 16 & 16,8 & 17,5 \\
\hline 17 & 18,3 & 16 \\
\hline 18 & 22,1 & 19,9 \\
\hline 19 & 21,3 & 17,4 \\
\hline 20 & 16,5 & 18,7 \\
\hline 21 & 17,1 & 17,9 \\
\hline 22 & 17,9 & 16,7 \\
\hline 23 & 19,23 & 16,2 \\
\hline 24 & 16,8 & 20,7 \\
\hline 25 & 18,96 & 18 \\
\hline 26 & 17,3 & 19,2 \\
\hline 27 & 23 & 19,6 \\
\hline 28 & 17,3 & 19 \\
\hline 29 & 15,6 & 19,9 \\
\hline 30 & 21,2 & 18,5 \\
\hline Média & 18,36 & 17,91 \\
\hline Desvio Padrão & 2,09 & 1,59 \\
\hline
\end{tabular}




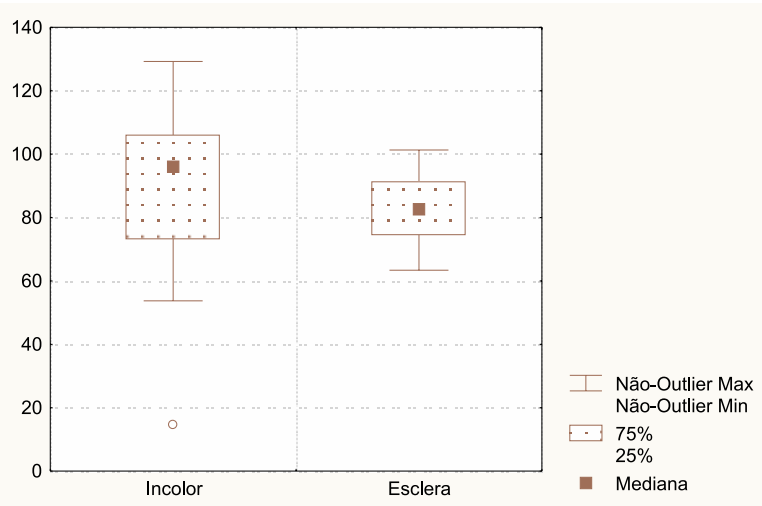

Figura 5 - Comparação entre as médias de resistência ao impacto dos grupos.

Os resultados foram submetidos a análise de variância pelo teste " $t$ " de Student. Não houve diferença estatística $(p>0,05)$ tanto para a dureza $(p$ $=0,35)$ como para a resistência ao impacto $(\mathrm{p}=$ $0,44)$ entre as duas resinas.

\section{Dıscussão}

O que se observou perante os resultados obtidos neste trabalho, foi uma maior dureza Knoop média para a resina incolor, embora não tenha sido observado diferença estatística entre os dois materiais $(\mathrm{p}>0,05)$. Os valores encontrados estão dentro das médias estabelecidas na literatura para dureza superficial de resinas termopolimerizáveis para base de dentaduras ${ }^{1,3,5,6,8,10}$. Essa proximidade de resultados ocorreu devido à provável semelhança na composição química das resinas, apesar do acréscimo de corante na resina para esclera, que não afetou significativamente a dureza superficial. Todavia, observou-se diferença entre a manipulação dos materiais estudados, a resina para esclera apresentou uma consistência mais espessa, mesmo nos momentos iniciais da mistura, dando a impressão que a proporção pó/líquido recomendada pelo fabricante apresentava quantidade de líquido insuficiente. Assim, o processo inicial de mistura até chegar à fase borrachoide ocorreu em menos tempo que para a resina incolor.

No que se refere à resistência ao impacto, a resina incolor apresentou uma média superior à resina para esclera, embora a diferença encontrada não

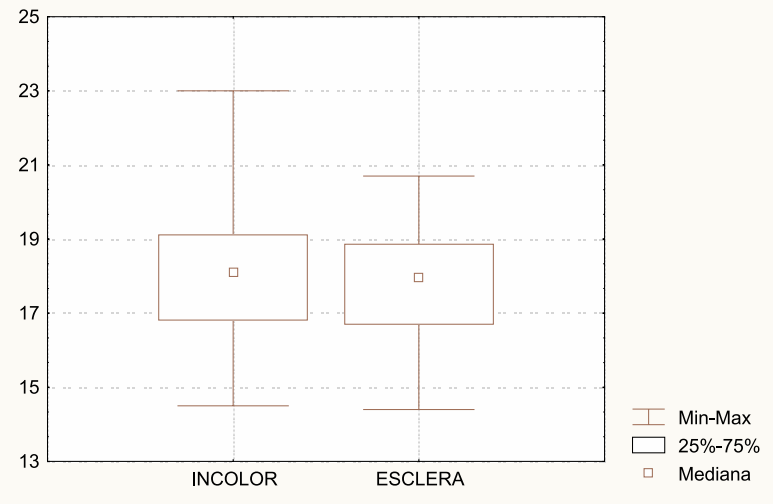

Figura 6 - Comparação entre as médias de dureza knoop dos grupos de resina.

tenha sido estatisticamente significante $(\mathrm{p}>0,05)$. Os valores são similares aos encontrados na literatura para resinas termopolimerizáveis para base de dentaduras $1,3,6,8-10,12$. O valor apresentado para a amostra $\mathrm{n}^{\circ} 3$ da resina incolor foi bem inferior aos demais, provavelmente devido a alguma bolha que tenha se formado no interior desta, durante a prensagem, mas isso foi compensado pelos demais valores, não representando diferença significativa para a análise realizada. Essa similaridade de resultados ocorreu provavelmente devido à proximidade na composição química das resinas, sendo que o acréscimo do corante para esclera não afetou significativamente a resistência ao impacto. A não significância nos valores obtidos condiz que a pequena diferença nos resultados não traz também significância clínica, pois a prótese ocular sofre menos tensões do que uma prótese sujeita as tensões na mastigação.

\section{Conclusão}

1. O valor médio de dureza Knoop da resina para esclera foi de 17,9 e para a incolor foi de 18,3 .

2. O valor médio da resistência ao impacto foi de $82 \mathrm{~J} / \mathrm{m}$ para a resina para esclera e de 88 $\mathrm{J} / \mathrm{m}$ para a resina incolor.

3. Não houve diferença estatística $(p>0,05)$ tanto para a dureza $(\mathrm{p}=0,35)$ como para a resistência ao impacto $(\mathrm{p}=0,44)$ entre as duas resinas. 


\section{Agradecimentos}

À disciplina de Prótese Parcial Removível do Departamento de Prótese e Periodontia da Facul-

\section{REFERÊNCIA BIBLIOGRÁFICA}

1. ANUSAVICE, K.J. et al. Phillips' science of dental materials 10 ed. Philadelphia:W.B. Saunders, 1996.

2. CARLOS, N.B.; HARRISON, A. The effect of untreated UHMWPE beads on some properties of acrylic resin denture base material. J Dent, v. 25, n.1, p. 59-64, 1997.

3. CRAIG, R. G. et al. Restorative dental materials. 10 ed., Mosby, St. Louis:Mosby, 1999.

4. DiAZ ARNOLD, A. M.; DUNNE, J. T.; JONES, A. H.; Microhardness of provisional fixed prosthodontic materials. J Prosthet Dent., v. 82, n.5, p.525-8, Nov. 1999

5. DONAVAN, T. E.; HURST, R. G.; CAMPAGNI, W. V. Physical properties of acrylic resin polymerized by four different techniques. J Prosthet Dent, v.54, n.4, p.522-4, Oct. 1985 .

6. HARRISON, A.; HUGGET, R.; JAGGER, R.C. The effect of a cross-linking agent on the abrasion resistance and impact strength of an acrylic resin denture base material. J Dent, v. 6, n.4, p. 299-304, 1978 dade de Odontologia de Piracicaba e ao AMR do Centro Técnico Aeroespacial pela permissão de uso dos aparelhos utilizados nas medições.

7. HONOREZ, P.et al. The effect of three processing cycles on some physical and chemical properties of a heat-cured acrylic resin. $\mathbf{J}$ Prosthet Dent, v.61, n.4, p.510-7, Apr. 1989.

8. NEIHART, T.R.; LI, S.H.; FLINTON, R.J. Measuring fracture toughness of high-impact poly(methyl methacrylate) with the short rod method. J Prosthet Dent, v. 60, n. 2, p.249-53, 1988

9. PRICE, C.A. The effect of cross-linking agents on the impact resistance of a linear poly(methyl methacrylate) denture-base polymer. J Dent Res., v. 65, n. 7, p. 987-92, 1986.

10. ROBINSON, J. G.; McCABE, J. F.; Impact strenght of acrylic resin denture base materials with surface defects. Dent Mater, v.9, n.6, p.355-60, Nov. 1993.

11. RODE, R. Prótese ocular ôca em resina acrílica: contribuição para a melhoria da mobilidade. 1968. 58f. Tese (doutorado) Faculdade de Odontologia de São José dos Campos - Universidade Estadual Paulista. São José dos Campos.

12. VALliTtU, P. K.; VOJTKOVA, H.; LASSILA, V. P.; Impact strenght of denture polymethyl methacrylate reinforced with continuous glass fibers or metal wire. Acta Odontol Scand, v.53, n.6, p.392-6, Dec. 1995. 